\title{
Multiple Gas Rings in SA Galaxies
}

\author{
J. Palouš and B. Jungwiert ${ }^{1}$ \\ Astronomical Institute, Academy of Sciences of the Czech Republic, \\ Bočni II, 14131 Prague 4, Czech Republic
}

\section{Introduction}

Rings are frequently observed in barred galaxies (e.g. Buta \& Crocker 1993). Their origin is attributed to gas accumulation near resonances, which is due to a combination of the gravitational torques of a bar component acting on inelastically colliding clouds: outer rings are presumably associated with the Outer Lindblad Resonance (OLR), inner rings with the 4/1 resonance, and nuclear rings with the Inner Lindblad Resonance (ILR).

However, multiple rings are also observed in normal non-barred, or not obviously barred galaxies (e.g. NGC 7217, Verdes-Montenegro et al. 1995) so that we may ask what is the mechanism of their formation. We argue in this contribution that even a very weak bar or a triaxial distortion in the galaxy center, which would hardly be identified from observations, leads to long-lasting ring-like distributions of gaseous clouds. The formation time-scales of rings associated with different resonances are similar in our model so that all types of rings can coexist in galaxies with a high degree of axial symmetry in their central parts, such as SA galaxies.

\section{N-body simulations}

An N-body sticky-particle code, described in detail by Palouš et al. (1993) and Jungwiert \& Palouš (1995; hereafter Paper I), was used to follow the ensemble of 8000 gas clouds in a weakly barred potential (maximum tangential force of the bar less than $3 \%$ of the axisymmetric force).

A time evolution of the initially axisymmetric disk is shown in Figure 1 for the bar angular speed $\Omega_{b a r}=30 \mathrm{~km} \mathrm{~s}^{-1} \mathrm{kpc}^{-1}$ and a rotation curve nearly flat $\left(\sim 200 \mathrm{~km} \mathrm{~s}^{-1}\right.$ ) beyond $R \sim 1 \mathrm{kpc}$. After $\sim 1$ Gyr a nuclear ring emerges in the central region while two large-scale spiral arms develop outside the corotation (CR). They evolve into a double-ring configuration under the action of the bar torques: at the transient stage from the spiral to the ring-like morphology $(\sim 2$ Gyr) the two outer rings touch one another near the bar minor axis but they separate later on $(\sim 2.5 \mathrm{Gyr})$ and both remain conspicuous until the end of the simulation (5 Gyr, i.e. $\sim 20$ bar rotations). An inner ring (well defined at $\sim 2$ $\mathrm{Gyr}$ ) and another inner pseudoring (never complete over $360^{\circ}$ but with segments apparent at $3 \mathrm{Gyr}$ ) exist between the ILR and CR.

\footnotetext{
${ }^{1}$ Observatoire de Meudon, 5 Place Jules Janssen, 92190 Meudon, France
} 

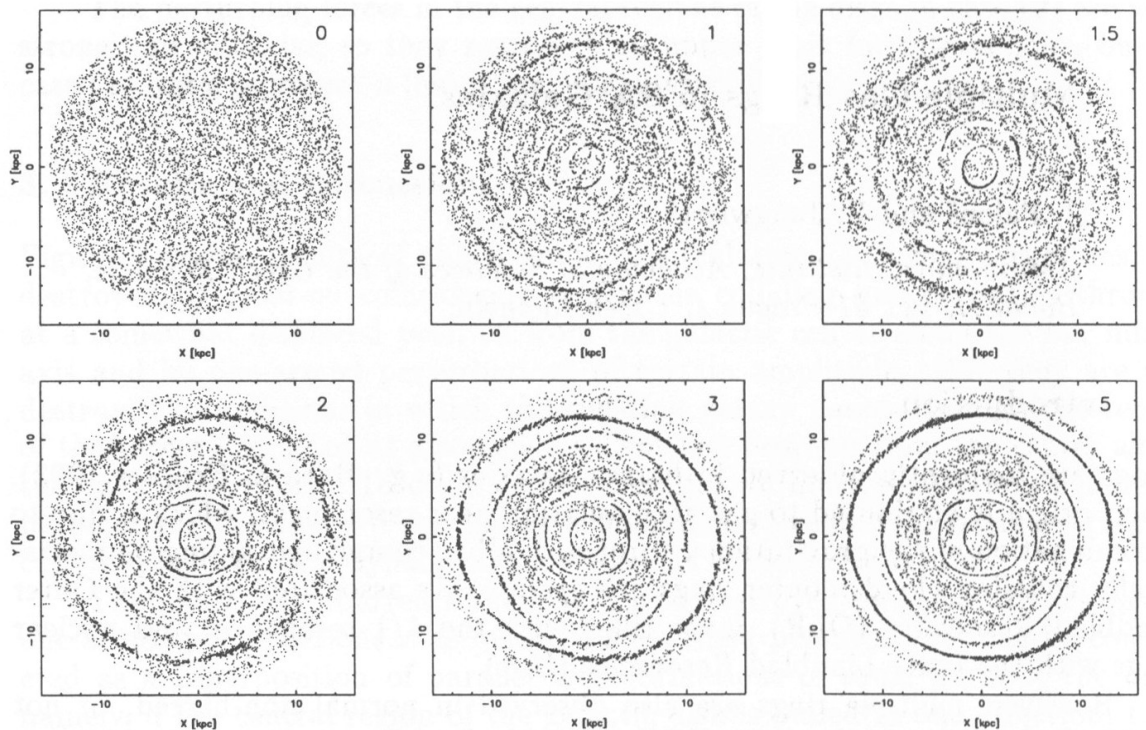

Figure 1. Evolution of the gaseous disk. Nuclear, inner and two outer rings appear. A fragment of a $6 / 1$ pseudoring is present as well. The outermost band of clouds is not a genuine ring: it is a consequence of the initial truncation of the disk at $15 \mathrm{kpc}$. The bar major axis is horizontal. The number in the upper right corner gives the time in Gyr.

Table 1 gives the sizes and formation time-scales for the rings as well as the resonances with which they are associated.

\section{Multiple Rings and Double Outer Rings}

The formation of rings connected to the ILR, 4/1 and OLR resonances was recognized in previous sticky-particles models (Schwarz 1981, 1984; Combes \& Gerin 1985). Some questions related to the ring morphology are however still open: mechanism for ring creation in unbarred galaxies, simultaneous formation of triple rings, observations of double outer rings.

Table 1. Sizes and formation times of rings.

\begin{tabular}{crrr} 
Resonance & Major axis [kpc] & Axial ratio & Formation time [Gyr] \\
\hline ILR & 2.0 & 0.85 & 1.5 \\
$4 / 1$ & 4.2 & 0.93 & 2 \\
$6 / 1$ & 5.6 & 0.95 & $>5$ \\
OLR & 10.8 & 0.93 & 2.5 \\
OLR & 13.4 & 0.93 & 2.5 \\
\hline
\end{tabular}


We have presented simulations which account for all of the three issues. A weak oval distortion that might not be recognized through observations is sufficient to generate multiple rings. The combination of two outer rings, one perpendicular and the other parallel to the bar (located slightly inside and outside the OLR, respectively), is long-lived. Such a combination is observed (Buta \& Crocker 1991) but only one of the outer rings could persist in models of Schwarz (1981).

The absolute times for ring formation (Table 1) are not of great importance since they depend on the details of the model (bar properties, degree of inelasticity of collisions, cloud cross sections) but their ratio has more significance: nuclear, inner and outer rings evolve on comparable timescales, differing by less than an order of magnitude.

An additional outcome of our experiment is the formation of a pseudoring near the $6 / 1$ resonance that was not recognized in previous works. We show in Paper I that for lower bar speeds (when the region between the ILR and CR is broader) a full 6/1 ring may exist. We suggest that the hexagonal shape of some observed inner rings, noted by de Vaucouleurs (1963), could be linked to the importance of the $6 / 1$ resonance.

The appearance of rings connected to higher order resonances is related to the low velocity dispersion in the system of colliding clouds. The question of the ring resistance against the gravity perturbations is addressed in Paper I together with limits of the model validity.

In contrast to our experiments, no galaxies are observed to have more than three rings or pseudorings. More than three rings could be a non-physical feature of the simulations, however it would be interesting to try to search observationally for such a possibility. Rings or pseudorings connected to higher order resonances (like 6/1) are expected to be much less robust than the main resonance rings and could have been unrecognized so far.

Acknowledgments. We are grateful to F. Combes and P. O. Lindblad for numerous discussions.

\section{References}

Buta, R. \& Crocker, D. 1991, AJ, 102, 1715

Buta, R. \& Crocker, D. 1993, AJ, 105, 1344

Combes, F. \& Gerin, M. 1985, A\&A, 150, 327

de Vaucouleurs, G. 1963, ApJS, 8, 31

Jungwiert, B. \& Palouš, J. 1995, A\&A, submitted

Palouš, J., Jungwiert, B., \& Kopecký, J. 1993, A\&A, 274, 189

Schwarz, M. P. 1981, ApJ, 247, 77

Schwarz, M. P. 1984, MNRAS, 209, 93

Verdes-Montenegro, L., Bosma, A., \& Athanassoula, E. 1995, A\&A, 300, 65 